\title{
Aplicações Clínicas do Peptídeo Natriurético do Tipo B
}

\section{Clinical Applications of B-Type Natriuretic Peptide Assays}

\author{
Humberto Villacorta Júnior e Evandro Tinoco Mesquita \\ Hospital Pró-Cardíaco - Rio de Janeiro, RJ
}

\section{Fisiologia do sistema PePtídeo NATRIURÉTICO}

O sistema peptídeo natriurético é ativado sempre que o coração sofre uma agressão, seja ela crônica seja aguda, na tentativa de compensar os sistemas vasoconstritores que são ativados nessas situações. As principais ações desse sistema são a promoção de diurese e vasodilatação. Sabe-se, no entanto, que uma série de efeitos adicionais é deflagrada com a liberação desses peptídeos, como inibição do sistema renina-angiotensina-aldosterona, inibição simpática, inibição de crescimento de células musculares lisas e possivelmente redução de apoptose ${ }^{1}$.

Três peptídeos são conhecidos - o peptídeo do tipo A (liberado pelos átrios), o do tipo B (liberado pelos ventrículos) e o do tipo $C$, liberado por células vasculares. O peptídeo do tipo B (BNP), por ser liberado diretamente pelos ventrículos, é o que melhor reflete a situação cardíaca, tendo alcançado maior aplicabilidade clínica. O estimulo para sua liberação é o estiramento das fibras ventriculares causado por uma sobrecarga de pressão ou de volume ${ }^{1,2}$. Mais recentemente demonstrou-se que isquemia miocárdica, levando a aumento de tensão parietal por disfunção sistólica e/ou diastólica, também pode promover liberação de $\mathrm{BNP}^{3}$.

Antes de sua liberação na circulação, o BNP apresenta precursores. No interior do miócito, o pro-BNP é clivado e libera na circulação duas moléculas - o BNP, que é a fração ativa, e a fração N-Terminal-pro-BNP (NT-proBNP), que é inativa, mas que também pode ser dosada no sangue.

Esses peptídeos agem através da ligação com receptores específicos, do tipo A e B. A degradação e eliminação são feitas através da ligação com receptores do tipo $C$, e por ação de uma enzima chamada endopeptidase neutra.

Em indivíduos normais, o BNP é influenciado por idade e sexo, sendo mais elevado nos idosos e nas mulheres quando comparados a homens da mesma faixa etária ${ }^{4}$.

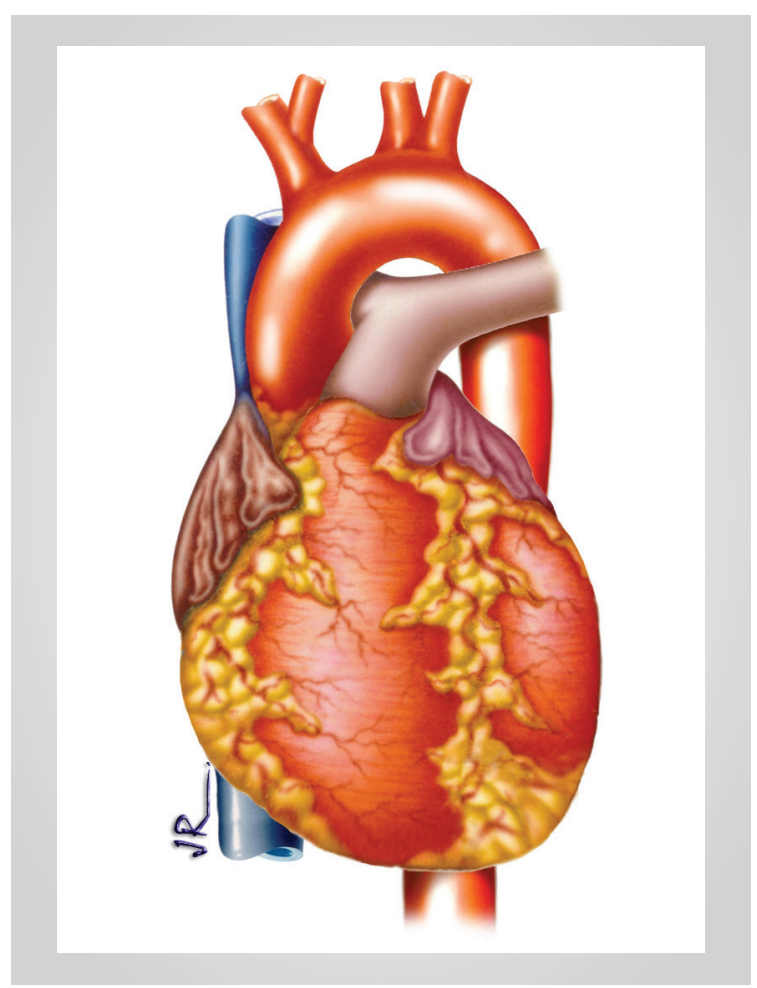

\section{TÉCNICAS DE DOSAgem do BNP}

As primeiras técnicas desenvolvidas para dosagem do BNP eram complicadas e demoradas, o que dificultava sua utilização, principalmente em situações de emergência. 0 desenvolvimento de testes de dosagem rápida (sistema point of care) possibilitou a utilização desse marcador bioquímico em uma grande variedade de situações na prática clínica. 0 primeiro e, até o momento, único teste por sistema point of care foi desenvolvido em San Diego, Estados Unidos e está disponível no Brasil desde 2001. Também já está disponível no Brasil um teste para dosagem do NT-pro-BNP, embora não seja por sistema point of care.

O BNP é dosado por imunofluorescência. Devem-se coletar $5 \mathrm{ml}$ de sangue em tubo contendo EDTA. Uma gota 
é colocada na tira e introduzida no aparelho, obtendo-se resultado em quinze minutos. O BNP é estável por pelo menos quatro horas à temperatura ambiente, por até dois dias refrigerado $\left(4-8^{\circ} \mathrm{C}\right)$ e por pelo menos doze meses quando estocado a $-20^{\circ} \mathrm{C}$.

\section{INSUFICIÊNCIA CARDÍACA}

A maior aplicação do BNP, até o momento, é no diagnóstico e manuseio de pacientes com insuficiência cardíaca (IC). Comentamos a seguir algumas situações em que o BNP pode ser útil nessa síndrome.

\section{Diagnóstico de disfunção sistólica de VE}

Estudos populacionais mostram utilidade do BNP como exame de triagem para o diagnóstico de disfunção sistólica de VE. Em um estudo escocês, BNP maior que $17,9 \mathrm{pg} / \mathrm{ml}$ apresentou sensibilidade de $76 \%$ e especificidade de $87 \%$ em detectar disfunção ventricular grave (fração de ejeção menor que 30\%). Em indivíduos acima de 65 anos a acurácia foi ainda maior ${ }^{5}$.

Em nosso meio, Ribeiro e cols. ${ }^{6}$ estudaram a capacidade do BNP em detectar disfunção sistólica de VE em pacientes portadores de doença de Chagas. Naqueles com eletrocardiograma ou radiografia de tórax alterados, um valor de BNP acima de 60,7 pg/ml apresentou sensibilidade e valor preditivo positivo (VPP) de $80 \%$ e especificidade e valor preditivo negativo (VPN) de $97 \%$ em detectar disfunção sistólica6 .

\section{Diagnóstico e prognóstico ambulatorial da IC}

O diagnóstico ambulatorial da IC por vezes pode oferecer dificuldades, principalmente em ambientes de atendimento primário. De fato, em vários estudos populacionais, menos de $40 \%$ dos pacientes com suspeita de IC feita pelo médico generalista tiveram esse diagnóstico confirmado, quando avaliados por cardiologistas $^{7}$. No Hillindon Heart Failure Study ${ }^{8}$ realizado em Londres, de 122 pacientes encaminhados pelo médico do atendimento primário para uma clínica especializada, 35 (29\%) tiveram o diagnóstico de IC confirmado. O desempenho do BNP no diagnóstico de IC nessa população foi muito bom (usando a curva ROC, a área sob a curva foi de 0,96). Com um valor de corte de 76,4 pg/ml, obteve-se um elevado VPN (98\%), com aceitável VPP (70\%). A sensibilidade e a especificidade foram, respectivamente, $97 \%$ e $84 \%$. Os resultados desse estudo serão validados em um estudo multicêntrico (The UK Natriuretic Peptide Diagnosis Study).

Em relação ao prognóstico, um estudo avaliou 290 pacientes consecutivos que foram submetidos a cateterismo cardíaco por disfunção sistólica de VE assintomática ou levemente sintomática ${ }^{9}$. Em um seguimento de 27 meses, 24 pacientes faleceram de causa cardíaca e 25 foram hospitalizados por IC ou infarto agudo do miocárdio (IAM). Os níveis de BNP medidos na ocasião do cateterismo correlacionaram-se de modo independente tanto com mortalidade cardíaca quanto com eventos cardíacos (morte somada a hospitalizações). Houve aumento de $4 \%$ no risco, para cada aumento de $10 \mathrm{pg} / \mathrm{ml}$ nos níveis de BNP.

Outro estudo avaliou pacientes com IC de recente começo, sendo estudados um subgrupo de 108 pacientes que faziam parte de um estudo populacional de pacientes diagnosticados pela primeira vez com IC, em Bromley, Londres $^{10}$. BNP, NT-pro-BNP e NT-pro-ANP foram medidos no momento em que o paciente foi visto pela primeira vez, antes, portanto, da estabilização clínica. No seguimento de 38 meses, quarenta pacientes morreram, sendo 34 de causas cardiovasculares. Os três peptídeos foram fortes preditores de mortalidade (área sob a curva 0,70, 0,73 e 0,68, respectivamente).

\section{Monitorização terapêutica}

Vem-se estudando também sua utilidade para guiar a terapêutica, fazendo-se a progressão das doses de medicamentos guiada pelo BNP. Um estudo piloto mostrou que a taxa de eventos cardiovasculares foi menor no grupo guiado por BNP, comparado ao grupo tratado convencionalmente ${ }^{11}$. Um estudo multicêntrico está em andamento para avaliar essa questão.

\section{Diagnóstico hospitalar da IC}

A situação em que o valor do BNP está mais sedimentado é no manuseio de pacientes com IC hospitalizados. Esses pacientes apresentam níveis elevados de BNP, o que facilita sua discriminação em relação a pacientes sem IC. Em relação a diagnóstico, o maior estudo foi o realizado por Maisel e cols., em que foram avaliados 1.538 pacientes com dispnéia aguda atendidos em unidades de emergência ${ }^{12,13}$. Nesse estudo multicêntrico, os pacientes com IC puderam ser facilmente diferenciados daqueles com outros diagnóstico. Os valores medianos de BNP no grupo com IC e sem IC foram, respectivamente, 675 \pm 65 versus $110 \pm 33 \mathrm{pg} / \mathrm{ml}$. A dosagem de BNP foi superior a dois critérios clínicos previamente utilizados para o diagnóstico de IC (Framingham e Nhale) e mostrou boa capacidade para excluir o diagnóstico de IC (VPN de $90 \%$ ). A sensibilidade e especificidade para um corte de $100 \mathrm{pg} / \mathrm{ml}$ foi de $90 \%$ e $73 \%$ ).

Em nossa instituição, a dosagem de BNP foi introduzida na prática clínica em abril de 2001. No mesmo período, foi iniciado um protocolo para estudar a utilidade do BNP no diagnóstico e prognóstico de pacientes com IC. Os dados sobre os primeiros setenta pacientes que chegaram à unidade de emergência com dispnéia aguda já foram publicados em $2002^{14}$. Os níveis de BNP em pacientes com IC e sem IC foram, respectivamente, $990 \pm 550$ e $80 \pm 67$ pg/ml. Um corte de 200 pg/ml apresentou sensibilidade, especificidade, VPP e VPN, 
respectivamente, de $100 \%$, 97,1\%, 97,3\% e 100\%.

No estudo Redhot ${ }^{15}$, em que foram avaliados pacientes com IC descompensada na unidade de emergência, observou-se uma falta de correlação entre o que o plantonista da unidade de emergência classificava como paciente grave e a evolução desse paciente. A utilização do BNP mostrou melhor correlação com a evolução do que a impressão do plantonista isoladamente. No estudo Basel, demonstrou-se que a utilização do BNP em pacientes com dispnéia aguda agiliza o diagnóstico ou exclusão da IC, resultando em menor tempo de internação e menor custo $^{16}$.

\section{Diagnóstico da IC diastólica}

Maisel e cols. avaliaram 294 indivíduos encaminhados para realização de ecocardiograma ${ }^{17}$. Os pacientes com disfunção diastólica no ecocardiograma apresentaram valores médios de BNP de $286 \pm 31 \mathrm{pg} / \mathrm{ml}$ contra 33 $\pm 3 \mathrm{pg} / \mathrm{ml}$ naqueles com função normal. Pacientes com o padrão restritivo apresentaram os maiores valores de BNP $(408 \pm 66 \mathrm{pg} / \mathrm{ml})$ e aqueles sintomáticos tinham valores altos de BNP independentemente do tipo de padrão de enchimento diastólico. A área sob a curva para detectar qualquer tipo de disfunção diastólica foi 0,92 e um corte de $62 \mathrm{pg} / \mathrm{ml}$ mostrou sensibilidade de 85\%, especificidade de $83 \%$ e acurácia de $84 \%$.

No estudo multicêntrico BNP Trial ${ }^{18}$, um corte de BNP de $100 \mathrm{pg} / \mathrm{ml}$ apresentou sensibilidade de 86\%, VPN de $96 \%$ e acurácia de $75 \%$ para detectar disfunção diastólica. Em casuística em nossa instituição, os pacientes com IC e disfunção sistólica de VE apresentaram maiores níveis de BNP que aqueles com função sistólica preservada ( $1.180 \pm 330$ versus $753 \pm 228$ pg/ml, p = 0,029), mas foi possível diferenciar o paciente com IC e função sistólica preservada daquele sem IC, em que os valores médios de BNP foram $753 \pm 228$ e $89 \pm 28$ respectivamente $(p<0,001)^{19}$.

Em um estudo populacional, no entanto, o BNP não foi um bom preditor de disfunção diastólica pré-clínica, sugerindo que nos pacientes com disfunção diastólica, apenas aqueles com disfunção mais acentuada ou com IC clínica manifesta podem ser detectados com o uso do BNP20.

\section{Prognóstico de pacientes hospitalizados por IC}

Em um estudo do Hospital dos veteranos, em San Diego, tanto o BNP de internação quanto sua variação durante a hospitalização foram preditores de evolução ${ }^{21}$. Pacientes cujos valores de BNP reduziram durante a internação tiveram o melhor prognóstico. Aqueles em que não houve variação do BNP apresentaram maior taxa de reinternação hospitalar em trinta dias, e aqueles com elevação do BNP tiveram maior mortalidade hospitalar e em até trinta dias de seguimento. Em nossa instituição, pacientes com BNP na internação maior que 760 pg/ml apresentaram maior taxa de mortalidade ou reinternação em trinta dias. A sensibilidade e a especificidade para predizer eventos em pacientes com BNP acima desse corte foram de $72 \%$ e $69 \%$, respectivamente ${ }^{22}$.

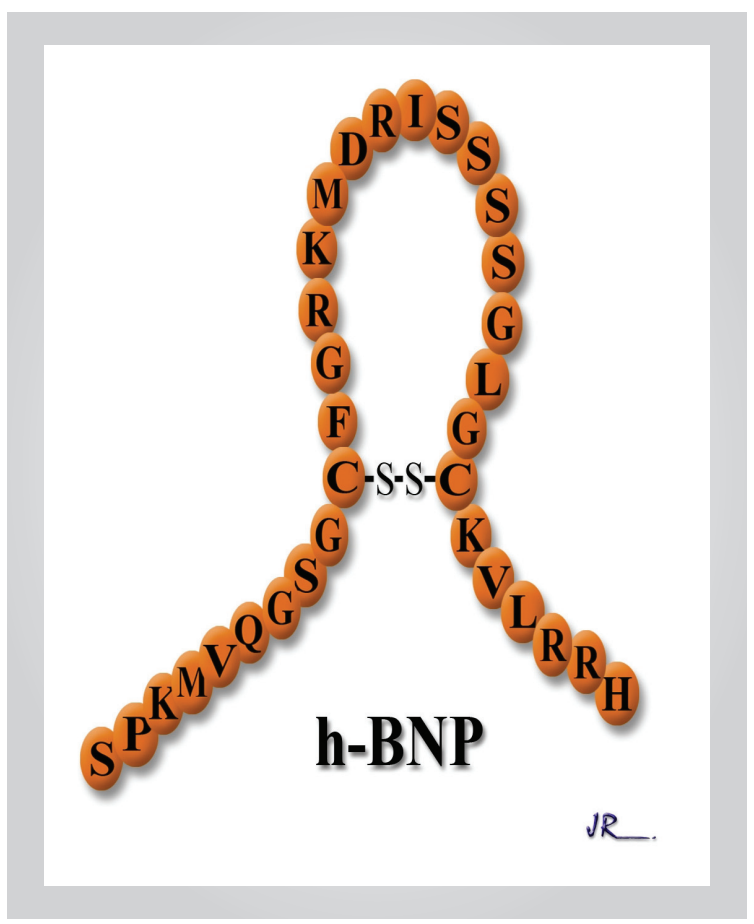

\section{SíNDROMES CORONARIANAS AGUDAS}

O BNP vem se mostrando importante marcador prognóstico em pacientes com síndromes coronariana aguda, tanto naqueles com IAM com supradesnivelamento de ST quanto em pacientes com IAM sem supradesnivelamento ou angina instável ${ }^{23-25}$. No maior estudo realizado nessa área, o BNP basal dosado em pacientes IAM com ou sem supradesnivelamento de ST e em angina instável correlacionou-se com a mortalidade e com o risco de desenvolver IC ou IAM em trinta dias e aos dez meses. A razão de chances para mortalidade aos dez meses, para o segundo, terceiro e quarto quartis de BNP, foi, respectivamente, de 3,8, 4,0 e 5,8.

Nosso grupo estudou o valor do BNP na internação em 631 pacientes atendidos com dor torácica em nossa unidade de emergência, no diagnóstico de IAM sem supradesnivelamento de $\mathrm{ST}^{26}$. Os pacientes com supradesnivelamento de ST foram excluídos. No momento da chegada à unidade de emergência eram dosados BNP, troponina I e CKMB-massa. Após três e nove horas eram repetidas as dosagens de troponina e CKMB-massa. 0 diagnóstico final de IAM baseou-se na positividade em qualquer momento, da troponina ou da CKMB-massa. Procuramos avaliar se a dosagem do BNP na internação do paciente com dor torácica aumentava a acurácia do diagnóstico de IAM ao longo da internação na unidade de emergência. A média dos valores de BNP nos pacientes com IAM, angina instável e com outros diagnósticos que não insuficiência coronariana aguda foi, respectivamente, 
$200 \mathrm{pg} / \mathrm{ml}, 78 \mathrm{pg} / \mathrm{ml}$ e $28 \mathrm{pg} / \mathrm{ml}$. A sensibilidade da CKMB-massa, troponina, BNP e com os três marcadores juntos foi de $46 \%, 51 \%, 71 \%$ e $87 \%$, respectivamente ( $p<0,001$ para comparação de BNP isoladamente e todos os marcadores juntos em relação a CKMB-massa e troponina). O valor preditivo negativo para os respectivos marcadores foram 93\%, 93\%, 95\% e 98\% ( $p=0,0016$ para comparação entre todos os marcadores juntos em relação a CKMB-massa e troponina). Por meio de análise multivariada, os preditores independentes de IAM foram infradesnivelamento de ST (razão de chances de 6,0), BNP $>100 \mathrm{pg} / \mathrm{ml}(R C=2,3)$, diabetes $(R C=2,5)$, CKMB-massa $>5(\mathrm{RC}=11,9)$ e troponina $>1,0(\mathrm{RC}$ $=36,6)$. Esses dados sugerem que a adição do BNP aos marcadores convencionais melhora a sensibilidade e o VPN do diagnóstico do IAM em pacientes sem supradesnivelamento de ST.

\section{Preditor de morte por Qualquer CAUSA EM ESTUDOS POPULACIONAIS}

Um grande estudo populacional, com 3.346 pacientes, publicado recentemente, procurou estabelecer a relação entre o BNP e o NT-pro-ANP com o risco para morte por qualquer causa, assim como a relação com o risco de desenvolver um evento cardiovascular, IC, fibrilação atrial, acidente vascular encefálico ou ataque isquêmico transitório e doença arterial coronariana ${ }^{27}$. Após um seguimento de 5,2 anos, 119 indivíduos faleceram e 79 tiveram um primeiro evento cardiovascular. Após ajuste para os fatores de risco cardiovascular, cada aumento de um desvio padrão nos valores logarítmicos de BNP esteve associado a aumento de $27 \%$ no risco de morte por qualquer causa, $28 \%$ no risco de um primeiro evento cardiovascular, $77 \%$ no risco de desenvolver IC, 66\% no risco de desenvolver FA, e um aumento de $53 \%$ no risco de apresentar um acidente vascular encefálico ou ataque isquêmico transitório. Não houve relação com o desenvolvimento de eventos relacionados a doença arterial coronariana. Resultados semelhantes foram observados com o NT-pro-ANP.

\section{Preditor de morte súbita (auxílio na INDICAÇÃO DO DESFIBRILADOR CARDÍACO)}

Em pacientes com IC, a dosagem do BNP pode identificar os pacientes de maior risco para desenvolver morte súbita, podendo vir a fazer parte da avaliação desses pacientes quanto à indicação de desfibrilador. Em um estudo austríaco ${ }^{28}$, somente $1 \%$ dos pacientes com valores logarítmicos de BNP abaixo de 2,11 apresentou morte súbita, comparado com $19 \%$ naqueles com valores acima desse limite. Nesse estudo foram avaliados 452 indivíduos. Em três anos, 89 pacientes faleceram, sendo 44 por morte súbita. A dosagem de BNP foi o único preditor independente de morte súbita. 0 mesmo grupo está organizando um estudo multicêntrico para comprovar esses achados.

\section{EstenOSE AÓRTICA}

Em portadores de estenose aórtica, os valores de BNP estão elevados naqueles com sintomas ${ }^{29}$. Uma vez que a presença de sintomas é um dos fatores para avaliação da indicação de cirurgia nesses pacientes, o BNP poderia ser incorporado a essa avaliação auxiliando na indicação cirúrgica.

\section{EMBOLIA PULMONAR}

O ventrículo direito, quando sob estresse, pode produzir BNP, embora em quantidade menor que o ventrículo esquerdo ${ }^{1}$. Pacientes com embolia pulmonar, principalmente aqueles com disfunção de ventrículo direito (VD), apresentam alguma elevação de BNP. Em pacientes atendidos em unidade de emergência com dispnéia e diagnóstico final de embolia pulmonar, esses valores usualmente variam de 100 a $400 \mathrm{pg} / \mathrm{ml}^{12,14}$. Nos pacientes com embolia pulmonar, os valores de BNP correlacionam-se com a presença de disfunção de VD e são preditores de pior evolução ${ }^{30,31}$.

\section{Hipertensão pUlmonar PRIMÁRIA}

Em um estudo com sessenta pacientes portadores de hipertensão pulmonar primária, o BNP foi um preditor independente de mortalidade ${ }^{32}$. Os pacientes foram tratados com prostaciclina. Três meses após o diagnóstico, dosou-se novamente o BNP. Nos sobreviventes, em um seguimento de 24 meses, observou-se queda do BNP entre as duas medidas ( $217 \pm 38$ versus $149 \pm 30$ ), enquanto nos pacientes que faleceram houve aumento do BNP (365 \pm 77 versus $544 \pm 68$ ). A sobrevida foi extremamente baixa para pacientes com BNP > $180 \mathrm{pg} / \mathrm{ml}$.

\section{SínCOPE}

Tanimoto e cols. ${ }^{33}$ estudaram 148 pacientes consecutivos com síncope: 61 desses pacientes tiveram síncope de etiologia cardíaca. Usando um corte de 40 $\mathrm{pg} / \mathrm{ml}$, a dosagem de BNP apresentou sensibilidade de $82 \%$ e especificidade de $92 \%$ em identificar pacientes com etiologia cardíaca para a síncope.

\section{Conclusões}

A dosagem do BNP vem ganhando grande número de possíveis utilidades na prática clínica. Desde sua aplicação inicial, de identificar disfunção sistólica de VE, inúmeras outras situações cardiovasculares foram acrescentadas à lista de doenças possíveis de ser manuseadas com o uso de BNP. Sua utilidade em pacientes com IC está bem estabelecida. Nas outras enfermidades cardiovasculares, cada dia mais evidências se acumulam, sugerindo que, num futuro próximo, o BNP possa ser acrescentado ao manuseio desses pacientes. 


\section{REFERÊNCIAS}

1. Cowie MR and Mendez GF. BNP and Congestive Heart Failure. Curr Probl Cardiol 2003; 44: 264-311.

2. Nakagawa $\mathrm{O}$, Ogawa $\mathrm{Y}$, Itoh $\mathrm{H}$ et al. Rapid transcriptional activation and early mRNA turnover of brain natriuretic peptide in cardiocyte hypertrophy: evidence for brain natriuretic peptide as an "emergency" cardiac hormone against ventricular overload. J Clin Invest 1995; 96: 1280-7.

3. Stein BC and Levin RI. Natriuretic peptides: physiology, therapeutic potential, and risk stratification in ischemic heart disease. Am Heart J 1998; 135: 914-23.

4. Redfield MM, Rodeheffer RJ, Jacobsen SJ, Mahoney DW, Bailey KR, Burnett JC. Plasma brain natriuretic peptide concentration: impact of age and gender. J Am Coll Cardiol 2002; 40: 976-82.

5. McDonagh T, Robb SD, Murdoch DR et al. Biochemical detection of left-ventricular systolic dysfunction. Lancet 1998; 351: 9-13.

6. Ribeiro AL, dos Reis AM, Barros MV et al. Brain natriuretic peptide and left ventricular dysfunction in Chagas'disease. Lancet 2002; 360: 461-2.

7. Remes J, Miettinen H, Reunanen A et al. Validity of clinical diagnosis of heart failure in primary health care. Eur Heart J 1991; 12: 315-21.

8. Cowie MR, Struthers AD, Wood DA et al. Value of natriuretic peptides in assessment of patients with possible new heart failure in primary care. Lancet 1997; 350: 1349-51.

9. Tsutamoto T, Wada A, Maeda K et al. Plasma brain natriuretic peptide level as a biochemical marker of morbidity and mortality in patients with asymptomatic or minimally symptomatic left ventricular dysfunction. Eur Heart J 1999; 20: 1799-807.

10. Cowie MR, Fox KF, Wood DA et al. N-terminal Pro-BNP predicts prognosis in patients presenting with heart failure for the first time better than does N-terminal Pro-ANP or BNP. Circulation 2000; 102: 781:5.

11. Troughton RW, Frampton CM, Yandle TG et al. Treatment of heart failure guided by plasma aminoterminal brain natriuretic peptide $(\mathrm{N}$ BNP) concentrations. Lancet 2000; 355: 1126-30.

12. Maisel AS, Krishnawami P, Nowak RM et al. Rapid measurement of B-type natriuretic peptide in the emergency diagnosis of heart failure. N Engl J Med 2002; 347: 161-7.

13. McCullough PA, Nowak RM, McCord J et al. B-type natriuretic peptide and clinical judgement in emergency diagnosis of heart failure: analysis from Breathing Not Properly (BNP) Multinational Study. Circulation 2002; 106: 416-22.

14. Villacorta H, Duarte A, Duarte NM et al. Valor do peptídeo natriurético do tipo B no diagnóstico da insuficiência cardíaca congestiva em pacientes atendidos com dispnéia na unidade de emergência. Arq Bras Cardiol 2002; 79: 564-8.

15. Maisel A, Hollander JE, Guss D et al. Primary results of the Rapid Emergency Department Heart Failure Outpatient Trial (REDHOT): a multicenter study of b-type natriuretic peptide levels, emergency department decision making, and outcomes in patients presenting with shortness of breath. J Am Coll Cardiol 2004; 44: 1328-33.

16. Mueller C, Scholer A, Laule-Kilian K et al. Use of B-type natriuretic peptide in the evaluation and management of acute dyspnea. New Engl J Med 2004; 350: 647-54.

17. Lubien E, DeMaria A, Krishnawamy P et al. Utility of B-type natriuretic peptide in detecting diastolic dysfunction: comparison with Doppler velocity recordings. Circulation 2002; 105: 595-601.

18. Maisel AS, McCord J, Nowak RM et al. Bedside B-type natriuretic peptide in the emergency diagnosis of heart failure with reduced or preserved ejection fraction: results from the Breathing Not Properly Multinational Study. J Am Coll Cardiol 2003; 41: 2010-7.

19. Villacorta H, Campos A, Duarte N, Martins MV, Mesquita ET. Utility of B-type natriuretic peptide in the diagnosis of heart failure with preserved systolic function in patients presenting to the emergency department with dyspnea. J Cardiac Fail 2002; 8 (4): S28.

20. Redfield MM, Rodeheffer RJ, Jacobsen SJ et al. Plasma brain natriuretic peptide to detect pre-clinical ventricular systolic or diastolic dysfunction: a community-based study. Circulation 2004; 109: 3176-82.

21. Cheng V, Kazanegra R, Garcia A et al. A rapid bedside test for B-Type natriuretic peptide predicts treatment outcomes in patients admitted for decompensated heart failure: a pilot study. J Am Coll Cardiol 2001; 37: 386-91.

22. Villacorta H, Martins MV, Mesquita ET, Dohmann HJF. Admissional B-type natriuretic peptide is an independent predictor of outcome in patients with decompensated heart failure. J Cardiac Fail 2003; 9 (5): S38.

23. Arakawa $\mathrm{N}$, Nakamura M, Aoki H, Hiramori K. Plasma brain natriuretic peptide concentrations predict survival after myocardial infarction. J Am Coll Cardiol 1996; 27: 1656-61.

24. De Lemos JA, Morow DA, Bentley JH et al. The prognostic value of B-type natriuretic peptide in patients with acute coronary syndromes. N Engl J Med 2001; 345: 1014-21.

25. White HD, French JK. Use of brain natriuretic peptide levels for risk assessment in non-ST-elevation acute coronary syndromes. J Am Coll Cardiol 2003; 42: 1909-16.

26. Bassan R, Potsch A, Maisel et al. B-type natriuretic peptide: a novel early blood marker of acute myocardial infarction in patients with chest pain and no ST segment elevation. European Heart Journal 2005; 26 : 234-40.

27. Wang TJ, Larson MG, Levy D et al. Plasma natriuretic peptide levels and the risk of cardiovascular events and death. N Engl J Med 2004; 350: 655-63.

28. Berger R, Huelsman M, Strecker K et al. B-type natriuretic peptide predicts sudden death in patients with chronic heart failure. Circulation 2002; 105: 2392-7.

29. Gerber IL, Stewart RA, Legget ME et al. Increased plasma natriuretic peptide levels reflect symptom onset in aortic stenosis. Circulation 2003; 107: 1884-90.

30. ten Wolde M, Tulevski LL, Mulder JW et al. Brain natriuretic peptide as a predictor or adverse outcome in patients with pulmonary embolism. Circulation 2003; 107: 2082-4.

31. Kruger S, Graf J, Merx MW et al. Brain natriuretic peptide predicts right heart failure in patients with acute pulmonary embolism. Am Heart J 2004; 147: 60-5.

32. Nagaya N, Nishikimi T, Uematsu M et al. Plasma brain natriuretic peptide as a prognostic indicator in patients with primary pulmonary hypertension. Circulation 2000; 102: 865-70.

33. Tanimoto K, Yukiiri K, Mizushige K et al. Usefulness of brain natriuretic peptide as a marker for separating cardiac and noncardiac causes of syncope. Am J Cardiol 2004; 93: 228-30 